\section{COSMIC RAYS}

\section{Backing for Muon Theory}

A NEW analysis of the spectrum of muons from cosmic rays at energies of more than $1,000 \mathrm{GeV}$ has given added support to the notion that muons may be produced by way of a special "X process" involving a short lived intermediate particle. This idea was first put forward by H. E. Bergeson et al. (Phys. Rev. Lett., $19,1487 ; 1967)$ to explain the unusual angular distribution of muons from cosmic rays detected in an experiment in Utah.

Bergeson et al. contended that the measured angular distributio 1 was not consistent with the muons' being solely the byproduct of pions and kaons produced in the upper atmosphere. Some other process had to be invoked, and they suggested that the solution lay in what they called an $\mathrm{X}$ process, for which the variation with energy of muon production was flatter than for production from pions and kaons. One interesting possibility was that the $\mathrm{X}$ was in fact the intermediate boson, which is held to mediate the weak interaction but has so far eluded detection. Another view was that an integral charge SU3 triplet was responsible for the $\mathrm{X}$ process.

Bergeson et al. pointed out that the spectrum derived from a combination of muons from pions and kaons with those from the $\mathrm{X}$ process give-with one exception-a convincing fit to other cosmic ray data between $10^{11}$ and $10^{14} \mathrm{eV}$, and could also account for the existence of mu-less air showers at $10^{15} \mathrm{eV}$. There has. however, been some opposition to this scheme, given fuel by the need to assume that the photonuclear cross-section increases at energies of more than $1,000 \mathrm{GeV}$ to five times the value that it has at normal accelerator energies, about $20 \mathrm{GeV}$.

P. Kiraly and A. W. Wolfendale (Phys. Lett., 32, $510 ; 1970)$ have shown that the presence of an $\mathrm{X}$ process need not depend on a large rise in cross-section. The weak link in the reasoning, they suggest, lies in the fact that the Osborne-Wolfendale-Palmer spectrum, which was the previously accepted muon scheme, was not measured directly by Bergeson et al. in this energy range but rested on the correlation of vertical cosmic ray intensity with depth underground and an assumed rate of energy loss. Kiraly and Wolfendale claim that if a higher rate of energy loss is taken the need for such a large cross-section is removed.

In the original analysis the $\mathrm{X}$ particles were assumed to have the same form of production spectrum as the pions and the vertical muon spectrum was derived from the angular distribution of the data. Kiraly and Wolfendale have followed recent practice by taking a larger constant in the energy loss formula. They also used the evidence from gamma ray experiments of a lower limit to the number of muons at sea level produced from pions. By then making the reasonable assumption that the number of muons produced by the $\mathrm{X}$ process is about 1.5 per cent of that from pions they show that the number of muons from pions at sea level can be made consistent with the evidence from gamma rays. This means that a big increase in photonuclear cross-section need not be a precondition of the existence of the $\mathrm{X}$ process. They also point out that the assumption of Bergeson et al. that the pion spectrum has the same slope as the primary spectrum is not necessarily true.

\section{MATERIALS}

\section{Special Ceramics}

\section{from a Correspondent}

RAPID advances in modern technology, for example in the nuclear power and gas turbine industries, are creating increasing demands for special materials with exceptional properties. Ceramists are turning to silicon nitride, silicon carbide and alumina, in that order, according to Dr N. F. Astbury, director of the British Ceramic Research Association, who gave the opening address at the Fifth International Symposium on Special Ceramics held in Stoke-on-Trent from July 14 to 16 . Other papers at the conference dealt with pyrolytic materials, the fabrication of ceramic bodies and the determination of the physical and chemical properties of a wide range of materials.

E. Voice (Atomic Energy Establishment, Winfrith) described the deposition of pyrolytic silicon carbide from methyltrichlorosilane-hydrogen mixtures in a fluidized bed of carbon-coated nuclear fuel particles. $\mathrm{He}$ showed that the temperature and gas composition are critical for the production of a fully dense impermeable layer. The morphological aspects of such layers were discussed by D. E. Y. Walker (UK Atomic Energy Authority, Springfields Works), and J. Ashford and J. C. Hodgson (Central Electricity Generating Board, Berkeley) described a technique for determining the mechanical behaviour of the layers under static and cyclic stress.

Because of the difference in thermal expansion it has been impossible to obtain strong vacuum tight metal-silica seals without using "graded silica-glass" components. L. Verheyden, K. Klein and H. Kind (Euratom, Italy) showed that silica can now be joined directly to 'Kovar' (54 per cent Fe: 28 per cent $\mathrm{Ni}$ : 18 per cent $\mathrm{Co}$ ) by using a composite wire with a core cf titanium surrounded by a 72 per cent Ag : 28 per cent $\mathrm{Cu}$ alloy.

The fourth and final session of the symposium was devoted solely to s.licon nitride ceramics. P. Griereson with K. H. Jack and S. Wild (University of Newcastle upon Tyne) described techniques whereby, at a given temperature, the nitrogen and oxygen partial pressure required to form silicon nitrides and oxynitrides is accurately determined, showing that $\alpha$ and $\beta$ are not low- and high-temperature forms but are "high oxygen potential" and "low oxyg€n potential" modifications: D. J. Godfrey working with M. W. Lindley (Admiralty Materials Laboratory, Poo'e) showed that reinforcement with carbon fibres or tungsten wire improves the toughness of silicon nitride tubes and increases the fracture stress. A novel technique for producing cellular silicon nitride was described by T. W. Lindop (Doulton Industrial Products). This low density. material has many potential applications in the nuclear reactor and refractory industries. The results of an investigation by S. Wild, P. Grieveson and $\mathrm{K}$. H. Jack (University of Newcastle upon Tyne) and N. J. Latimer (The Plessey Company) on the effect of magnesia additions on the hot-pressing behaviour of silicon nitride account for a number of previously unexplained features. At high temperatures, a liquid of composition near to that of enstatite $\left(\mathrm{MgSiO}_{3}\right)$ is formed and this acts during pressing as a flux and plasticizing agent to allow complete densification of the compact. 